\title{
A Geomorphic Framework for the Analysis of Microplastics in Riverine Sediments
}

\author{
Jerry Miller ${ }^{1, * *}$ and Suzanne M. Orbock Miller ${ }^{2}$ \\ ${ }^{1}$ Department of Geosciences \& Natural Resources, Western Carolina University, Cullowhee, USA \\ ${ }^{2}$ Tuscola High School, Waynesville, NC, USA
}

\begin{abstract}
The wide-spread use and persistence of plastics in the environment have placed them on the list of significant emerging pollutants. In contrast to marine environments, the analysis of plastic debris, including microplastics (particles $<5 \mathrm{~mm}$ in maximum diameter), in freshwater systems is limited, and even fewer studies have examined microplastics in riverine sediments. Nonetheless, it has become clear that microplastics are now a ubiquitous component of riverine ecosystems and their distribution is dependent on anthropogenic inputs and the physical and chemical processes that control their transport, transformation, and deposition along the drainage network. In many ways, the transport and fate of microplastics will parallel that of other particulate matter that has been extensively studied for at least the last 50 years. Here, we briefly explore the application of a geomorphic approach to the assessment of sediment-contaminated rivers to the microplastic problem, and argue that future studies can significantly benefit by incorporating the principles of this approach into their analyses.
\end{abstract}

\section{Introduction}

The ability to mold synthetic polymers (plastics) into an infinite variety of shapes, combined with their versatile nature in terms of weight, strength, durability, melting point, and chemical reactivity have made them virtually indispensable in modern manufacturing. There are about 20 distinct groups of plastics that are extensively used in everything from cosmetic products and cleansers to clothing, to plumbing, to packaging and ropes, among a host of other products. The development of synthetic polymers began in the late 1800s [1], but it was not until the 1950s that plastics were produced on an industrial scale. Since then, plastic production has increased exponentially, reaching 359 million metric tons [2], and is expected to increase significantly in the coming years [3].

Unfortunately, plastics released to the environment represent a significant emerging pollutant found in atmospheric, terrestrial, freshwater and marine systems. Microplastics (MPs), in particular, have received considerable attention as a global pollutant. While the definition of what constitutes a MP is a topic of debate, the most widely used definition is any plastic item measuring $<5 \mathrm{~mm}$ in its maximum (long) dimension, a size that can be

\footnotetext{
* Corresponding author: jmiller@email.wcu.edu
} 
ingested by many species of aquatic, benthic and terrestrial biota. Included in this definition are nanoplastics, which are often defined as particles with a maximum dimension of $<100$ nanometres $(\mathrm{nm})$ [4]. There are two dominant sources of MPs. Primary plastics include plastic beads used in cleaners and cosmetic products, and manufactured pellets used as feedstock in plastic production. A small, but quantitatively significant, portion of these beads make their way through waste water treatment plants (WWTPs), along with fibers from washed clothing, where they may be released to the aquatic environment (Fig. 1a). Alternatively, microplastics can be produced by the weathering and degradation of larger plastic items, which are released to the environment from both point and non-point sources, including urban centers, industrial effluent, sewage amended agricultural soils, tires, and atmospherically deposited particles (aerosols), among others [5,6]. Plastics from secondary sources are thought to be responsible for most microplastics in marine environments [3,7],

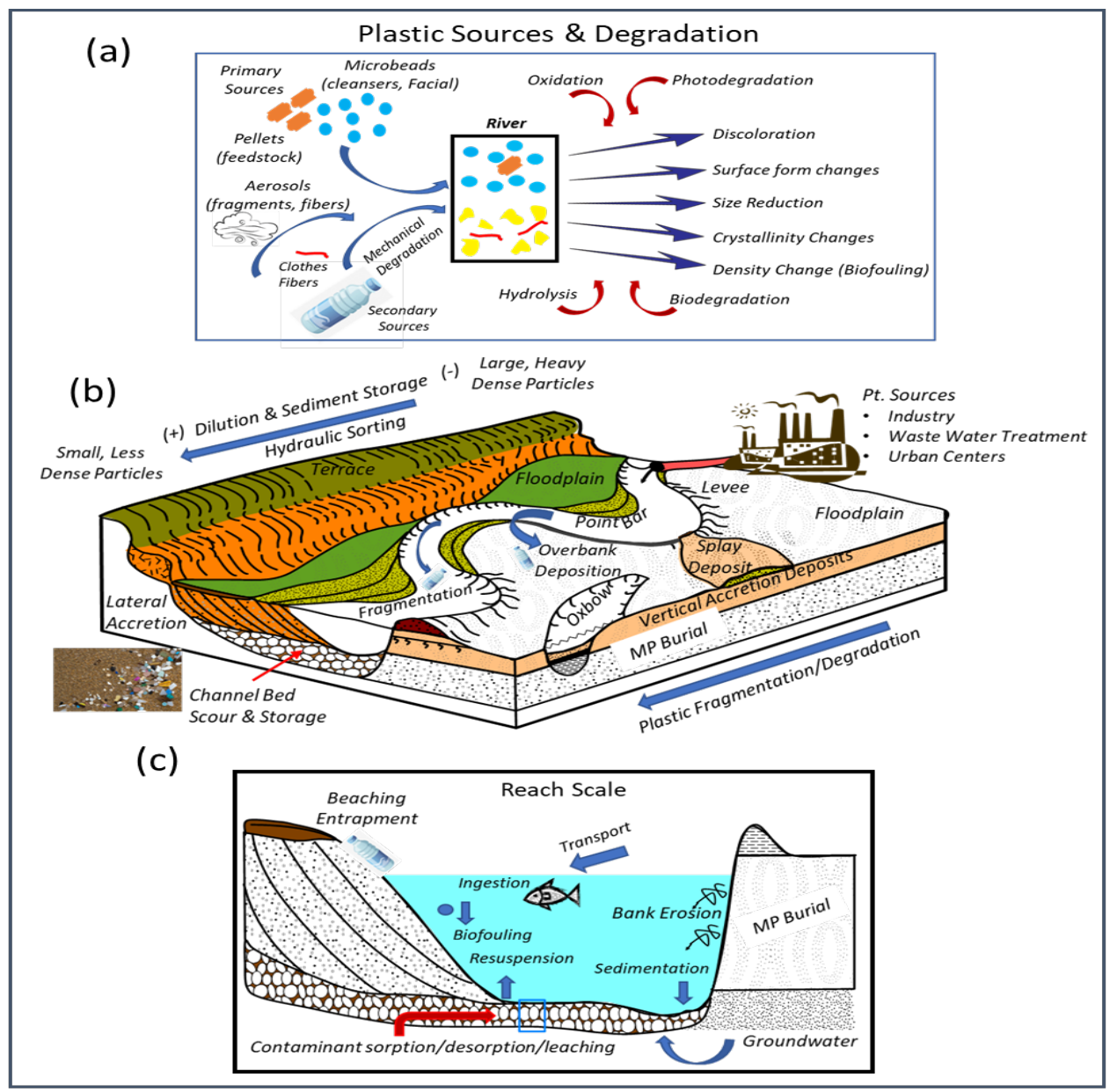

Fig. 1. (a) Schematic diagram of MP sources and degradation processes; (b) commonly observed floodplain depositional units and hydraulic partitioning processes; (c) common processes affecting MP in rivers.

and once modified by degradational processes (Fig. 1a), acquire a wide range sizes, textures and shapes of varying composition.

Our understanding of the effects of MPs on human and ecological health is currently limited, particularly within freshwater environments, and has generally focused on the unrealistically high concentrations of a limited number of forms [8]. Nevertheless, MPs possess traits suggestive of potentially significant ecological impacts, including (1) their 
ubiquitous distribution in atmospheric, marine, terrestrial and freshwater environments. Their abundance has been most extensively documented for oceanic waters, where modeling estimates suggest that there may be more than about 5 trillion particles, weighing between 7,000 [9] and more than 250,000 [10] metric tons floating in the world's oceans. Microplastics have also been found in every freshwater system studied; (2) they are highly durable, allowing MPs to remain in the environment for decades to hundreds of years [11]; (3) MPs can be ingested by a diverse range of organisms where they act as stressors that may damage organs, obstruct the gut and/or negatively affect hatching and growth rates $[4,12,13]$. There is also evidence that following ingestion, MPs may be transferred across trophic levels, allowing them to be bioaccumulated [14,15]; and (4) a wide range of chemical substances (plasticizers, antioxidants, stabilizers and colourants) are added to plastics during the manufacturing process to achieve their desired uses. They also have a high sorption potential, allowing them to serve as carriers (vectors) of organic and inorganic hydrophobic contaminants $[4,12,16,17]$, suggesting they may posing a risk to biota $[4,5,16,18]$.

Although several of the United Nations seventeen sustainable development goals (SDGs) indirectly address MPs, none of them do directly. Nonetheless, plastic debris is now almost universally considered an emerging pollutant that may have the potential to affect our ability to conserve biological diversity [3,19]. Given the potential impacts of MPs on ecosystem health, their sources, types, concentrations, transformations, and dispersal mechanisms need to be quantified, along with their potential impacts on biota. While the study of MPs is not new, the overwhelming majority date to the past decade and focus on marine environments [20]. Freshwater environments have received much less attention, and even fewer studies have focused on rivers and river sediments. The potential impacts of MPs on riverine ecosystems was largely missed by past monitoring and regulatory programs [14].

While there is a notable lack of data on MPs in rivers, the available data demonstrate that MP concentrations in alluvial sediments are similar to and, locally, exceed those in marine sediments [13,21]. In fact, Klein et al. [13] measured concentrations of MPs in the River Tame that reached 517,000 particles $\mathrm{m}^{-2}$, one of the highest abundances ever reported. These data suggest that in addition to the potential impacts of MPs on lotic ecosystems, rivers serve as an important sink and regulator of microplastic transport to marine environments; in fact, as much as $80 \%$ of plastic in the world's oceans may have originated from rivers $[22,23]$.

Recent studies of the distribution of MPs within riverine ecosystems show that their abundance is highly variable and dependent on anthropogenic inputs to the environment, and the physical and chemical processes that control their transport, transformation, and deposition along the drainage network. In many ways, the transport and fate of MPs will parallel that of other particulate matter that has been extensively studied for at least the last 50 years. Here, we briefly review the literature on MPs in river sediments, and explore the implications of previously conducted contaminated sediment studies to the analysis of MPs. In doing so, we highlight a few of the studies that are immediately needed to more fully understand the sources, transport and fate of plastics in riverine ecosystems.

\section{Key Traits of Microplastic Transport and Deposition in Rivers}

Conceptually, the transport of particulate matter in rivers is subjected to settling and sedimentation, transport by advective and dispersive forces, and particle resuspension from the channel bed and banks (Fig. 1), all of which depend on the density, size distribution, and shape of the particles $[3,24]$. Plastic debris in rivers differs from the more extensively studied mineral and rock fragments in all three of these properties. In terms of density and composition, plastics in river sediments are highly variable, but are typically dominated by the polymers that are most widely produced, including polyethylene (PE, $38 \%$ ), polypropylene (PP, $24 \%$ ), polystyrene (PS, 6\%) and polyvinyl chloride (PVC, 19\%) [6,13]. The density of these plastics range from $\sim 0.85->1.40 \mathrm{~g} \mathrm{~cm}^{-3}$, and possess densities less than 
most mineral and rock fragments $\left(\sim 2.65 \mathrm{~g} \mathrm{~cm}^{-3}\right)$. Moreover, while some polymer types are buoyant in freshwater, others are not. MPs also exhibit a broader range of particle sizes that when compared to natural particles of similar density (e.g., algae, woody debris, organic matter), are more durable and persistent [6]. The shapes of MPs also tend to be more variable, reflecting both the source of the plastics and their weathering and degradation (Fig. 1). Common shapes include fragments, fibers, beads, pellets, sheets, flakes and foams.

Birch et al. [25] argued that because of the diversity in the size, shape and density of MPs and the range of natural environmental conditions that exist, it is not possible to make generalized statements about their transport behavior in aquatic systems. Nonetheless, the low densities some plastics (e.g., PE, PP, PS) should allow for their widespread distribution in aqueous environments as they will float near the water surface until the particles are beached along the edge of the channel, are entrapped by vegetation, or are transported onto the floodplain during overbank events. Indeed, Cook et al. [26] used a novel method based on the transport of fluorescent dye and dyed microparticles composed of PE to assess the movement of neutrally buoyant MPs in the water column of a laboratory flume. Their experimental data found that low density, buoyant particles, followed theoretical dispersion theory and behaved in a manner similar to solutes, as expected. In contrast to these larger low-density, buoyant particles, high density plastics such as PVC and PET, which are also produced in large volumes, and smaller buoyant particles, are more susceptible to settling through the water column (Kooi et al., 2018) and may be deposited within the channel bed sediment [13].

Differences in polymer density may not be the only, or even dominate factor, controlling the deposition of MPs because their density may be increased by the development of biofilms on the particle surface (biofouling). The relatively rapid development of biofilms on suspended MPs has been observed in both marine [27] and freshwater [28] environments. Perhaps more importantly, biofilms increase the efficiency of forming aggregates of larger and denser particles [29]. These aggregates are often composed of small pieces of either buoyant or non-buoyant plastic incorporated into aggregated clusters of mineral matter (called heteroaggregates).

Interestingly, recent theoretically modeling suggests that the variations in polymer density on particle fate and retention were over-ridden by the effects of size changes imposed by aggregation [30,31]. Besseling et al. [30], for example, developed a theoretical transport model that found that the increased mass of suspended solids produced by heteroaggregation had a dramatic effect on the modeled fate and retention of MPs in rivers, and the location of the developed zones of MP accumulation (hot spots). The importance of aggregation on particle deposition is supported by the accumulation of MPs with a density both greater than and lower than water in alluvial and lacustrine sediments, showing that they can settle and become incorporated into freshwater deposits [13,32]. Given the importance of aggregation on microplastic transport and fate, the efficiency of the attachment between particles, and the factors that control it (e.g., biofilms, mineral composition and size, suspended sediment concentration, plastic composition, shape and texture, and degradation processes) are in need of study.

The accumulation of sediment-associated contaminants has demonstrated that both the concentration and mass of contaminants within the channel bed is dependent on the grain size, surface roughness and reworking of the channel bed sediments [33]. An especially important control is the erosion (scour) and fill of the channel bed during a flood. During erosion, contaminants are released to the water column, whereas subsequent deposition during the recessional phases of the event results sediment and contaminant accumulation in the bed materials. The reworked sediments are commonly referred to as the active layer, and the thickness and dynamics of the active layer significantly influence both the storage and transport rates of contaminants through time. The nature of the activite layer is controlled, in part, by sediment size, sediment supply, and the hydrologic conditions of the river. Armoring of the channel bed can significantly influence the frequency with which sediments are 
mobilized. A recent flume study by Oakelford et al. [34] showed that microplastic mobilization and storage was also linked to the dynamics of the active layer, which in turn was influenced in a complex way to the nature of the flood's hydrograph. Their data, then, suggest that the active layer may also control the accumulation and transport of MPs in rivers, and should be examined in more detail for natural systems. In fact, the few studies that have examined temporal differences in the MP transport and storage in river sediments show that concentrations and transport rates are highly susceptible to changes in flow conditions over a range of temporal scales [14,35]. For example, Hurley et al. [14] found that total microplastic contamination of channel bed sediments within the Irwell and Mersey catchments in the UK was reduced by an average of $64 \%$ and $81 \%$, respectively, following severe flooding in the winter of 2015/2016; the decrease at 18 of the 40 investigated sites was dramatic, reaching about an order of magnitude. At 7 of the sites, microbead contamination was completely removed, demonstrating that microplastic contamination could significantly change during a single event. Data collected along the Bloukrans River in South Africa demonstrated that MP concentrations may also change seasonally. In this case, higher concentrations were observed during the winter season, characterized by lower-flows, than during the wetter summer months [5].

The observed temporal changes described above are consistent with the hypothesis that higher flows mobilize sediments within the active layer and release MPs to the water column for transport (and vice versa). However, studies of sediment associated trace metals have shown that the thickness and dynamics of the active layer are not the only controls on contaminant accumulation in the channel bed. Concentrations are also dictated by the relative influx of contaminants to the river during runoff events. Depending on contaminant sources, concentrations may increase, particularly from urban sources when significant stores of contaminants and/or MPs are carried to the river by runoff. Once flushed to the river, these materials may be incorporated into the active layer of the channel bed. Alternatively, less contaminated sediments from diffuse sources may enter the river during floods, and dilute sediment-associated contaminants (and MPs) concentrations in the channel bed [36].

Studies of trace metal transport and fate have also shown that sediment-associated contaminants may be stored in the channel bed (and other alluvial deposits) as a result of channel bed aggradation [37,38] caused by channel instability. In this case, the contaminants are removed from active transport, but may be re-introduced years to centuries later during periods of channel incision. To our knowledge, no studies conducted to date have examined MP storage in aggrading rivers.

\section{Spatial Patterns in Contaminant and MP Concentrations}

The geomorphological-geochemical approach, formally defined by Macklin et al. [39], is commonly used for the assessment of rivers effected by hydrophobic contaminants. The approach assumes that the transport and fate of sediment-associated contaminants is governed in large part by physical (geomorphic) processes. Thus, their dispersal and depositional patterns can be assessed by analyzing the geomorphic processes responsible for particle transport and deposition, and the alluvial deposits that they form. We believe that the principles inherent in this approach are particularly appropriate for the assessment of MPs in alluvial sediments, and can be used to form a framework for the future analysis of MPs in alluvial (river) sediments.

A key finding of the geomorphological approach is that sediment-associated contaminants are not randomly distributed within the channel bed, but are partitioned into specific depositional features within the channel. As a result, at the reach scale, sedimentassociated contaminant concentrations vary spatially between channel bed features (e.g., pools, point bars, riffles, glides, etc.) as a function of their particle size, density, and shape as well as the topographic position, flow magnitude, and frequency of deposit inundation 
[37,40,41]. Given the range of compositions, sizes, densities, and shapes associated with MPs, we hypothesize that these reach-scale variations in plastic concentrations will be even more significant than those observed for hydrophobic contaminants associated with mineral matter. There are two important implications of this hypothesis. First, sampling sediments for MPs from different channel bed features may yield dramatically different results, even where the samples are located a few meters apart. As a result, comparison of MP concentrations along the river over significant distances, such as up- and downstream of a WWTP, may be more strongly controlled by the morphological channel unit sampled, than the input from a potential source, thereby, leading to erroneous interpretations. In fact, numerous publications have argued that more time, effort and money has been wasted on the analysis of sediment-associated contaminants as a result of poor sampling design, including the failure to understand the local variations in contaminant accumulation, than any other reason $[42,43]$. Second, given the time-consuming nature of the analysis of MPs in sediment, random sampling to assess statistically valid MP concentrations is unlikely to be feasible. Thus, for spatial analysis, it may be more appropriate to sample the same morphological unit at all sites along the river, than to collect multiple, statistically valid samples from the channel. In light of the above, there is a critical need to conduct well-designed analyses of the partitioning of MPs into discrete channel bed features in term of their abundance, size, density and composition, and to assess the relationship of microplastic characteristics to the sedimentology (grain size distribution, mineral composition, organic matter content) of the sampled units.

\section{Determination of MP Source(s)}

Spatial patterns in contaminant concentrations with channel bed sediments are commonly used to determine the source of the contaminants to the river. The most commonly observed trend is an abrupt increase in concentrations immediately downstream of a point source or source tributary, followed by a systematic downstream decline in concentrations from the observed peak. The downstream decrease in concentrations is due to: (1) hydraulic sorting (with larger, denser particles remaining closer to the source), (2) dilution associated with the dispersal of a finite amount of contaminants over a larger area, and by the introduction of uncontaminated sediments via tributary inputs and bank erosion, (3) contaminant storage in alluvial deposits, (4) biological uptake, and (5) chemical reactions [33,44-46].

Many of the studies conducted to date on the distribution of MPs in rivers have hypothesized that MPs will exhibit a predictable pattern in their concentration, composition, size, shape, and density as a function of the input from MP sources (e.g., urban centers, WWTP, and industries), the distance downstream from these point sources and variations in environmental conditions (e.g., channel morphology and hydraulic regime) that influence particle transport, transformations, and deposition $[3,8,13]$. The observed relations between microplastic source(s) and concentration in channel sediments has been mixed. McCormick et al. [47], for example, showed that microplastic concentrations increased downstream of WWTP (from Nel et al., 2018, pg. 951), whereas Mani et al. [48]found higher microplastic loads along the Rhine River in the vicinity of densely populated areas, and along river reaches draining populated and recreational areas. Similarly, Nel et al. [5] found that the extent of microplastic pollution along the Bloukrans River in South Africa was consistently higher within the boundary of Grahamstown than in areas removed from the city. In contrast, Klein et al. [13] found no significant correlation between population density and the mass and number of MPs in river sediments. They were also unable to find spatial trends in microplastic concentrations associated with WWTP, suggesting that plastic sources could not be determined using spatial concentration data.

Klein et al. [13] attributed the inability to link microplastic concentrations to suspected sources on variations in hydraulic conditions that controlled the transport and deposition of 
plastic particles. This is consistent with the contaminated sediment literature which has clearly demonstrated that hydrophobic contaminants may be concentrated in zones of preferential deposition [49,50]. Macklin and Lewin [45], for example, found that trace metal transport and fate varied between narrow, high gradient transport zones, and wide, lower gradient sedimentation zones. We hypothesize that such variations are likely to influence the transport of MPs as well. Current studies, in general, have not attempted to quantitatively link MP concentration, size, density and shape to the morphology and hydrology the river and river valley; such quantitative studies are critically needed.

In addition to assessing microplastic sources by spatial variations in concentration, several studies have noted a link between microplastic size, shape, and/or composition to plastic sources [8,51]. Allen et al. [51], for instance, found that MPs in rivers draining cites were dominated by fibers. In contrast, fragments, and to a lesser degree films and fibers were the primary shapes in remote catchments of the French Pyrenees. Microplastics in these latter areas were attributed to the atmospheric deposition of particles transported from populated areas. Klein et al. [13] found that fiber concentrations were unexpectedly low along the Rhine-Main river system given the large number of sewage treatment plants that existed, and the potential for the release of fibers from washed cloths. Most microplastic particles were spheres (microbeads), which they attributed to cleansers and cosmetics. Castaneda et al.[32] (and others) also noted that the physical and chemical composition of MPs in the Great Lakes drainage system were similar to that of microbeads associated with household products. In addition, they observed that the average size of MPs along the St. Lawrence River was smaller at sites receiving industrial or municipal effluent than at sites devoid of direct effluent inputs, suggesting that size could be used as a source indicator. Although these approaches appear promising, there is still much to be learned about the use of microplastic composition, size, and shape as a source indicator, particularly given the potential variations in particle transport and deposition along river systems, and their partitioning into specific depositional units.

In recent years, a particularly effective means of assessing the source of toxic trace metals in riverine environments has been the use of stable isotopes. Lead isotopes have been particularly effective at determining lead and associated trace metal sources in aquatic environments. A significant advantage of using isotopic approaches is that they can often be used to quantitatively estimate the relative contributions of the material from a specific source. Given the potential for plastics to sorb hydrophobic contaminants, it seems possible that MPs could obtain lead at the site of their production or release, and that lead isotopic fingerprinting could be used to quantitatively assess the source of the MPs. To date, the use isotopic approaches in rivers has yet to be explored.

\section{Floodplain Environments and Chronological Records of Plastic Pollution}

The partitioning of the sediment-associated contaminants into discrete depositional features as noted above is not limited to the channel bed. Partitioning also occurs within those deposits associated with floodplains and terraces (Fig. 1b). We hypothesize that MPs will be partitioned at this spatial scale was well, and argue that MPs in well-defined and dated alluvial stratigraphic units should be analyzed to assess the variations that occur in MPs concentrations, size, density, and composition. Although the abundance of MPs in floodplains have yet to be analyzed, the contaminated sediment literature suggests that floodplains will serve as both an important sink and source of MPs and, thus, act as a critical regulator of microplastic export to marine environments. In addition, as a critical interface between the aquatic and terrestrial environment and a site of extensive anthropogenic utilization, floodplains will serve as a critical zone of microplastic exposure to biota. Thus, these data are critical to understand the long-term residence time of MPs in river systems, to 
calibrating and verifying quantitative models of MP transport and fate, and for defining realistic MP concentrations and forms for ecotoxicological studies.

The contaminated sediment literature also demonstrates that floodplains contain a record of contaminant influx to the river, which may be used to assess pollutant sources, temporal variations in the influx, transport and accumulation of contaminants to rivers, and the natural rates of system recovery to aquatic pollution. The assumption inherent in these analyses is that alluvial deposits reflect the source(s) and concentrations of sediment-associated contaminants within the river at the time of deposition, an assumption for which no data currently exist for MPs. However, if the assumption holds for MPs, then the alluvial stratigraphic record could be deciphered and compared to documented variations in human activities within the basin to assess the potential anthropogenic sources of MPs to the riverine environment. In general, the development of an alluvial chronology of contamination involves the geomorphic and sedimentologic characterization of the alluvial deposits, the sampling and analysis of the deposits for the contaminant of interest, in this case MPs, a determination of the relatively ages of the deposits using sedimentologic principles, and the application of absolute dating methods to constrain the age of the deposits in "calendar" years [33,52-55].

While such contaminant histories can be developed using any undisturbed, continuous or semi-continuous sequence of alluvial sediments, the most detailed assessments have used overbank deposits. Overbank floodplain deposits are produced by the deposition of suspended sediments from floodwaters that inundate the floodplain surface, a process that results in layers of younger materials that bury older sediments.

The analysis of dated cores for the assessment of microplastic influx to rivers has not, to our knowledge, been conducted using floodplain deposits. However, a limited number of studies have been conducted on sediment cores extracted from coastal [56], continental shelf [57], and freshwater lake [58] systems, and have shown that the concentrations of MPs in sediments parallel the known increases in microplastic production and use [8]. These studies suggest that sediments serve as a reliable archive of plastic pollution [8], and that sediments may be used to document variations in microplastic inputs and sources to aquatic environments, including rivers. In fact, the appearance of plastic in sedimentary records has been proposed as a marker for the onset of the Anthropocene [8]. Given the persistence of plastics in the environment, and the general lack of long-term monitoring of plastic waste in rivers [59], the analysis of sedimentary archives may be particularly useful in retrospectively capturing the occurrence, usage, release rates and changing types of plastics through time [58].

\section{Conclusions}

The quantitative study of MPs in rivers is in its infancy; most studies have been conducted with the past 5 years. It took more than 2-decades to link the study of contaminated sediments to a sound geomorphological-geochemical approach, arguably hindering our understanding of hydrophobic contaminant behavior in riverine ecosystems. Future studies of MP in sediments should utilize a holistic, integrated approach to minimize cost and time, and effectively assess the transport and fate of MPs in rivers.

\section{References}

[1]. S. Lambert and M. Wagner, in Freshwater Microplastics: Emerging Environmental Contaminants/, edited by M. Wagner and Lambert, Scott (Springer International Publishing, Cham, Switzerland, 2017), pp. 1-24.

[2]. M. Garside, Plastic Industry Statistics and Facts. Http://www.statista.com/topics/5266/plastics-industry/ (2020).

[3]. D. Eerkes-Medrano and R. Thompson, in Microplastic Contamination in Aquatic Environments, edited by E. Y. Zeng (Elsevier, 2018), pp. 95-132. 
[4]. A. Lusher and J. Mendoza, Microplastics in Fisheries and Aquaculture: Status of Knowledge on the Occurrence and Implications for Aquatic Organisms and Food Safety (Food and Agriculture Organization of the United Nations, 2017).

[5]. H. A. Nel, T. Dalu, and R. J. Wasserman, Sci. Total Environ. 612, 950 (2018).

[6]. M. Kooi, E. Besseling, C. Kroeze, A. P. Van Wezel, and A. A. Koelmans, in The Handbook of Environmental Chemistry (Springer International Publishing, 2018), pp. $125-152$.

[7]. V. Hidalgo-Ruz, L. Gutow, R. C. Thompson, and M. Thiel, Environ. Sci. Technol. 46, 3060 (2012).

[8]. P. Yao, B. Zhou, Y. Lu, Y. Yin, Y. Zong, M.-T. Chen, and Z. O'Donnell, Quatern. Int. 519, 274 (2019).

[9]. A. Cózar, F. Echevarría, J. I. González-Gordillo, X. Irigoien, B. Úbeda, S. HernándezLeón, et al., Proc Natl Acad Sci USA 111, 10239 (2014).

[10]. M. Eriksen, L. C. M. Lebreton, H. S. Carson, M. Thiel, C. J. Moore, J. C. Borerro, F. Galgani, P. G. Ryan, and J. Reisser, PLOS ONE 9, 1 (2014).

[11]. M. Zbyszewski and P. L. Corcoran, Water, Air, \& Soil Pollution 220, 365 (2011).

[12]. M. Cole, P. Lindeque, C. Halsband, and T. S. Galloway, Mar. Pollut. Bull.62, 2588 (2011).

[13]. S. Klein, E. Worch, and T. P. Knepper, Environ. Sci. Technol. 49, 6070 (2015).

[14]. R. Hurley, J. Woodward, and J. J. Rothwell, Nature Geoscience 11, 251 (2018).

[15]. J. M. Wright and J. C. Chambers, Appl. Vegetation Sci. 5, 237 (2002).

[16]. E. L. Teuten, J. M. Saquing, D. R. U. Knappe, M. A. Barlaz, S. Jonsson, et al., Philosophical Transactions of the Royal Society B: Biological Sciences 364, 2027 (2009).

[17]. C. G. Avio, L. R. Cardelli, S. Gorbi, D. Pellegrini, and F. Regoli, Environ, Pollut. 227, 207 (2017).

[18]. A. A. Koelmans, N. H. Mohamed Nor, E. Hermsen, M. Kooi, S. M. Mintenig, and J. De France, Water Research 155, 410 (2019).

[19]. W. J. Sutherland, M. Clout, I. M. Côté, P. Daszak, M. H. Depledge, L. et al., Trends Ecol. Evol. 25, 1 (2010).

[20]. GESAMP, Sources, Fate, and Effects of Microplastics in the Marine Environment: A Global Assessment. (2015), p. 96.

[21]. A. Lechner, H. Keckeis, F. Lumesberger-Loisl, B. Zens, R. Krusch, M. Tritthart, M. Glas, and E. Schludermann, Environ. Pollut. 188, 177 (2014).

[22]. A. L. Andrady, Mar. Pollut. Bullet. 62, 1596 (2011).

[23]. A. A. Horton, A. Walton, D. J. Spurgeon, E. Lahive, and C. Svendsen, Sci. Total Environ. 586, 127 (2017).

[24]. A. Ballent, A. Purser, P. de Jesus Mendes, S. Pando, and L. Thomsen, Biogeoscience Discussions 9, 18755 (2012).

[25]. Q. T. Birch, P. M. Potter, P. X. Pinto, D. D. Dionysiou, and S. R. Al-Abed, Rev. Environ. Sci. and Biotech. 19, 275 (2020).

[26]. S. Cook, H.-L. Chan, S. Abolfathi, G. D. Bending, H. Schäfer, and J. M. Pearson, Water Res. 170, 115337 (2020).

[27]. F. M. C. Fazey and P. G. Ryan, Environ. Pollut. 210, 354 (2016).

[28]. K. Parrish and N. L. Fahrenfeld, Environ. Sci.: Water Res. Technol. 5, 495 (2019).

[29]. L. Van Cauwenberghe, A. Vanreusel, J. Mees, and C. R. Janssen, Environ. Pollut. 182, 495 (2013).

[30]. E. Besseling, J. T. K. Quik, M. Sun, and A. A. Koelmans, Environ. Pollut. 220, 540 (2017).

[31]. L. Nizzetto, G. Bussi, M. N. Futter, D. Butterfield, and P. G. Whitehead, Environ. Sci.: Proc. Imp. 18, 1050 (2016).

[32]. R. A. Castañeda, S. Avlijas, M. A. Simard, and A. Ricciardi, Can. J. Fish. Aquat. Sci. 71, 1767 (2014).

[33]. J. R. Miller and S. M. Orbock Miller, Contaminated Rivers: A GeomorphologicalGeochemical Approach to Site Assessment and Remediation (Springer Science \& Business Media, 2007).

[34]. A. Ockelford, A. Cundy, and J. E. Ebdon, Scientific Reports 10, 1865 (2020).

[35]. S. Veerasingam, M. Mugilarasan, R. Venkatachalapathy, and P. Vethamony, Marine Pollut. Bull. 109, 196 (2016). 
[36]. J. R. Miller, P. J. Lechler, G. Mackin, D. Germanoski, and L. F. Villarroel, Sci. Total Environ.384, 355 (2007).

[37]. K. A. Hudson-Edwards, M. G. Macklin, J. R. Miller, and P. J. Lechler, J. Geochem. Expl. 72, 229 (2001).

[38]. L. F. Villarroel, J. R. Miller, P. J. Lechler, and D. Germanoski, Environ. Geol. 51, 283 (2006).

[39]. M. G. Macklin, Hudson-Edwards, K.A., H. E. Jamison, P. Brewer, T. J. Coulthard, A. J. Howard, and V. H. Renenda, in Mine, Water, and Environment (Seville, Spain, 1999), pp. 271-278.

[40]. W. L. Graf, S. L. Clark, M. T. Kammerer, T. Lehman, K. Randall, and R. Schroeder, Catena 18, 567 (1991).

[41]. S. C. Ladd, W. A. Marcus, and S. Cherry, Environ. Geol. 36, 259 (1998).

[42]. L. H. Keith, Warren. Crummett, John. Deegan, R. A. Libby, J. K. Taylor, and George. Wentler, Anal. Chem. 55, 2210 (1983).

[43]. A. J. Horowitz, Primer on Sediment-Trace Element Chemistry (Lewis Publishers, 1991).

[44]. S. O. Helgen and J. N. Moore, Environ. Sci. Technol. 30, 129 (1996).

[45]. M. G. Macklin and J. Lewin, Earth Surf. Proc. and Land. 14, 233 (1989).

[46]. M. P. Taylor and R. G. H. Kesterton, Geomorphology 42, 311 (2002).

[47]. A. McCormick, T. J. Hoellein, S. A. Mason, J. Schluep, and J. J. Kelly, Environ. Sci. Technol. 48, 11863 (2014).

[48]. T. Mani, A. Hauk, U. Walter, and P. Burkhardt-Holm, Scientific Reports 5, 17988 (2015).

[49]. M. G. Macklin and R. B. Dowsett, Catena 16, 135 (1989).

[50]. M. G. Macklin, P. A. Brewer, K. A. Hudson-Edwards, G. Bird, T. J. Coulthard, I. A. Dennis, P. J. Lechler, J. R. Miller, and J. N. Turner, Geomorphology 79, 423 (2006).

[51]. H. E. Allen, C.-P. Huang, G. W. Bailey, and A. R. Bowers, Metal Speciation and Contamination of Soil (CRC Press, 1994).

[52]. M. G. Macklin and K. Klimek, Appl. Geogr. 12, 7 (1992).

[53]. T. Matys Grygar, J. Elznicova, Š. Tůmová, M. Famera, M. Balogh, and T. Kiss, Geomorphology 254, (2015).

[54]. T. Matys Grygar, T. Novakova, O. Bábek, J. Elznicova, and N. Vadinová, Robust Assessment of Moderate Heavy Metal Contamination Levels in Floodplain Sediments: A Case Study on the Jizera River, Czech Republic (2013).

[55]. E. Ferrand, F. Eyrolle, O. Radakovitch, M. Provansal, S. Dufour, C. Vella, G. Raccasi, and R. Gurriaran, Geochim. Cosmochim. Acta 82, 163 (2012).

[56]. K. A. Willis, R. Eriksen, C. Wilcox, and B. D. Hardesty, Frontiers Mar. Sci. 4, 419 (2017).

[57]. J. Martin, A. Lusher, R. C. Thompson, and A. Morley, Scientific Reports 7, 10772 (2017).

[58]. S. Turner, A. A. Horton, N. L. Rose, and C. Hall, J. Paleolimnol. 61, 449 (2019).

[59]. D. K. A. Barnes, F. Galgani, R. C. Thompson, and M. Barlaz, Philosophical Transactions of the Royal Society B: Biological Sciences 364, 1985 (2009). 